\title{
Towards Automatic Landmarking on 2.5D Face Range Images
}

\author{
Pui Suk Ting \\ Faculty of Computer Science and Information Technology \\ Universiti Malaysia Sarawak \\ 94300 Kota Samarahan \\ Sarawak, Malaysia \\ polly_sukting@hotmail.com
}

\author{
Jacey-Lynn Minoi \\ Faculty of Computer Science and Information Technology \\ Universiti Malaysia Sarawak \\ 94300 Kota Samarahan \\ Sarawak, Malaysia \\ jacey@ fit.unimas.my
}

\begin{abstract}
In this paper, we propose an algorithm to automatically landmark points on 2.5 -dimensional (2.5D) face images. We applied the Scale-invariant Feature Transform (SIFT) method to a new automatic landmarking method. Automatic landmarking has a number of added advantages over manual landmarking and it is more accurate and less time consuming especially if the dataset is large. We developed an interactive Graphical User Interface (GUI) tool to ease the visualization of the extract face features, which are scale and transformation invariant. The threshold values are then analyzed and generalized to best detect and extract important keypoints or/and regions of facial features. The results of the automatic extracted keypoint features are shown in this paper.
\end{abstract}

Keywords-Scale-invariant Feature Transform (SIFT), automatic landmarking, features extraction

\section{INTRODUCTION}

The human vision system can perceive features such as the edges, tips or corners of the object, without any difficulties. For example, a human is able to detect and recognize the eyes, the tip of the nose and/or the mouth of a person at first glance. However, a computer is unable to do such task easily and effortlessly. The human vision system and brain mechanisms that are responsible for the detection of features are so complex that despite the work of neurobiologists, mathematicians and computer scientists, it is still not possible to replicate facial detection accurately.

In this paper, we propose a method to find distinct features on a face. We aim to make the feature detection process automatic, whereby the landmark points could be used for face analysis, face registration and recognition. Unlike the conventional manual landmarking, whereby a human intervenes to locate facial features visually, automatic landmarking is able to locate such facial features without the intervention of a human. An automatic landmarking process can save time, especially when data sets are huge. In this research, we focus our automatic landmarking on $2.5 \mathrm{D}$ face images.

A face image contains complex features including a large degree of background and face variations, such as the identity of the person, facial expression, head pose, facial hair variability, age, gender, cluttered background and etc. Face images come with different format types, sizes, scales and rotation, which may lead to difficulties in automatic landmarking processes. Hence, we proposed to use the Scale-invariant Feature Transform (or SIFT) on feature extractions process. SIFT was first introduced by David Lowe [1] and it is an algorithm to detect and extract keypoints or local features in images. SIFT holds the advantage of being able to detect features even under changes in image scale, noise and illumination.

Over the last decades, 3D images have become popular due to the advancement of 3D sensor and camera technologies; alongside with 2.5D range images. Range images (2.5D) have a number of added advantages over 2D images. A $2.5 \mathrm{D}$ image is defined as a simplified threedimensional $(\mathrm{x}, \mathrm{y}, \mathrm{z})$ surface representation that contains at most one depth $(\mathrm{z})$ value for every point in the $(\mathrm{x}, \mathrm{y})$ plane [2]. One can think of a $2.5 \mathrm{D}$ image as a grey-scale image, where the black pixel corresponds to the background, while the white pixel represents the surface point that is nearest to the camera [3]. 2.5D face images enable depth perception and allow one to manipulate the image alike a 3D image. In addition to range data, colour perception on a face image is also possible. The information from the range image can be extracted to derive different features regions. Therefore, a 2.5D image is used as a dataset to define the keypoint descriptor by extracting the facial surface information.

We have developed an interactive Graphical User Interface (GUI) (as shown in Figure 1), which aims to visualize the processes in SIFT on range images and to allow manipulation of the extracted feature descriptors. 